\title{
037 LAVERED RESERVOIRS WITH CROSSFLOW AND GRAVITY EFFECTS - NUMERICAL STUDIES
}

G.B. SAVIOLI, M.S. BIDNER, P.M. JACOVKIS and M.J. COCCO Buenos Aires University, Laboratorio de Ingeniería de Reservorios, Facultad de Ingeniería, Pabellón Industrias, Ciudad Universitaria, 1428 Buenos Aires, Argentina

\begin{abstract}
The two dimensional flow of oil through heterogeneous and bounded reservoirs, including gravity effects, is rigorously solved by finite differences. A family of finite difference schemes that depend on one parameter $\theta$, which varies between 0 and 1 , is applied. The system of linear equations is solved by a method based on Taylor Series of Matrix Functions (TSMF). TSMF is compared with the traditional Alternate Direction Implicit (ADI) and Block Successive Over Relaxation (Block-SOR) methods. TSMF consumes less CPU time than ADI and Block-SOR, using appropriate values of $\theta$ and time step $\Delta t$.

The numerical simulator is then applied to study the drawdown response of a two layer reservoir with crossflow and gravity forces. The pressure response of crossflow reservoirs lies between the response of the equivalent single layer system and that of the isolated layer system. When the vertical pressure gradient is increased by gravity, the gap between the response of the equivalent single layer system and that of the isolated layer system is enlarged. The pressure curves keep similar shapes as those of the nil gravity case. When the vertical pressure gradient is decreased by gravity, the gap is lessened. However, the pressure curves may become inverted.
\end{abstract}

\section{INTRODUCTION}

Reservoirs are not homogeneous. The simplest model of heterogeneity is the stratified formation, validated by the deposition process. The stratified formation consists of many layers of different permeabilities, porosities and thicknesses. When the layers are separated by impermeable shales and they only communicate at the wellbore, the reservoir is commingled. When the layers communicate in the formation, influencing each other, the reservoir presents crossflow.

The pioneer studies ${ }^{1,2}$ on multilayered reservoirs appeared in the 60's. They established the early time and long time pressure response of a well draining a reservoir with interlayer crossflow. The early time response is similar to the response of the commingled reservoir. The long time response is given by the equivalent single layer (homogeneous) reservoir having the same volume, with permeability and porosity which are the weighted mean of those of the multilayered one. The pressure response of a commingled reservoir is described by analytical solutions found applying Laplace ${ }^{1}$ and Hankel ${ }^{2}$ transforms. More than twenty years later, Prijambodo et $\mathrm{al}^{3}$ analyzed the behavior of the transitional period. With that purpose they presented a multilayered $2 \mathrm{D}$ (radial and vertical) model for the flow of oil towards the well. The layers were assumed to be homogeneous and the gravity was neglected. Numerical solutions were obtained applying finite differences and an implicit scheme.

Our interest lies on the analysis of heterogeneities and their effect on well tests ${ }^{4}$. For that reason we put forward a model where the spatial variations of permeability and porosity and also gravity effects are considered $^{5}$. The model was solved by a family of finite difference schemes. The family depends on one parameter, $\theta$, which varies from 0 to 1 . The stability of the schemes was previously analyzed applying the matrix method that takes into account the boundary conditions ${ }^{6}$. The resulting system of linear equations was then solved by the Block-SOR method.

This paper deals with the understanding of well-test response in flow of oil of constant compressibility through bounded, layered reservoirs in the presence of gravity effects. For that purpose, the model above described has been applied. But the system of linear equations is solved by a method based on Taylor Series of Matrix functions (TSMF). This method is compared with the traditional ADI and Block-SOR. Our contributions are: (1) Application of TSMF method which consumes less CPU time than ADI and 
Block-SOR if adequate values of $\theta$ and time step are chosen; (2) Study of the influence of gravity on the draw-down response of a layered reservoir with crossflow. As far as we know, both contributions are new to the petroleum literature.

The future aim is to couple this numerical model with optimization techniques ${ }^{7,8,9}$ for automatic history matching of well test measurements. With that tool, we hope to be able to infer reservoir parameters for stochastic modeling.

\section{THEORY}

\section{2-D model}

A multilayer, cylindrical reservoir of thickness $H$, with heterogeneous permeability and porosity, contains oil of constant compressibility. It has a well located at its central axis that penetrates the formation completely. With these assumptions, a model in two dimensions, $(r, z)$ is obtained ${ }^{5,6}$

$\frac{1}{r} \frac{\partial}{\partial r}\left(r \frac{k_{r}(r, z)}{\mu B} \frac{\partial p}{\partial r}(r, z, t)\right)+\frac{\partial}{\partial z}\left(\frac{k_{z}(r, z)}{\mu B}\left(\frac{\partial p}{\partial z}(r, z, t)-g \rho\right)\right)+q(r, z, t)=\frac{\phi(r, z) c}{B} \frac{\partial p}{\partial t}(r, z, t)$.

where $q(r, z, \mathrm{t})=0$ in all domain but the well (boundary condition).

The initial condition is

$$
p(r, z, t=0)=p_{I N} .
$$

The boundary conditions at the well $\left(r=r_{w}\right)$ are:

* known total flow rate,

$$
q_{T}=\frac{2 \pi}{\mu B} \int_{0}^{H} k_{r}\left(r_{w}, z\right)\left(r \frac{\partial p}{\partial r}\right)_{r=r_{w}} d z
$$

*pressures related by gravity forces,

$$
p\left(r_{w}, z, t\right)=p_{w f}+\rho g z
$$

where $p_{w f}=p\left(r_{w}, z=0, t\right)$.

At reservoir boundaries (outer, top and bottom), the non-flow condition is imposed. By Darcy's law,

$$
\left.\frac{k_{r}(r, z)}{\mu}\left(\frac{\partial p}{\partial r}(r, z, t)\right)\right|_{r=r_{e}}=0 \quad,\left.\quad \frac{k_{z}(r, z)}{\mu}\left(\frac{\partial p}{\partial z}(r, z, t)-g \rho\right)\right|_{z=0, H}=0 .
$$

\section{Numerical Solution}

Let us define dimensionless variables

$$
x_{D}=\ln \frac{r}{r_{w}} \quad, \quad z_{D}=\frac{z}{H} \quad, \quad t_{D}=\frac{t}{\mu c}
$$

A family of finite difference schemes, depending upon the parameter $\theta$, is applied to numerically solve the model equations $(1-4)^{5,6}$. The space coordinates are discretized using a grid system $\left(x_{D i}, z_{D j}\right)=\left(i \Delta x,\left(j+\frac{1}{2}\right) \Delta z\right), 0 \leq i \leq n_{i} ; 0 \leq j \leq n_{j} ;$ time is discretized as $t_{D}^{n}=n \Delta t$. Pressure at the representative mesh point $\left(x_{D i}, z_{D j}, t_{D}^{n}\right)$ is denoted by $P_{i, j}^{n}$. Choosing a suitable order of unknowns and equations and operating with the resulting matrix ${ }^{5,6}$, the linear system of equations to be solved may be written as

$$
(I+\theta T) \boldsymbol{P}^{n+1}=(I-(1-\theta) T) \boldsymbol{P}^{n}+\psi,
$$

where matrix $T \in R^{\bar{N} X \bar{N}}\left(\bar{N}=\left(n_{j}+1\right) n_{i}+1\right)$ has the following structure

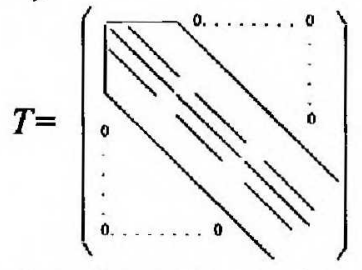

$(I+\theta T)$ is strictly diagonally dominant; therefore it is not singular and it follows that

$$
\boldsymbol{P}^{n+1}=(I+\theta T)^{-1}(I-(1-\theta) T) \boldsymbol{P}^{n}+(I+\theta T)^{-1} \psi \text {. }
$$


To solve Eq. (9), a method based on Taylor Series of Matrix Functions (TSMF) is introduced. Using $(1+\theta x)^{-1}=\sum_{n=0}^{\infty}(-1)^{n} \theta^{n} x^{n} \quad$ when $|\theta x|<1 \quad$, it follows that

$$
\boldsymbol{P}^{n+1}=\left(I-T+\theta T^{2}-\theta^{2} T^{3}+\theta^{3} T^{4}-\theta^{4} T^{5}+\ldots \ldots . .\right) \boldsymbol{P}^{n}+\gamma
$$

where $\gamma=(I+\theta T)^{-1} \psi$, if $\left|\theta \lambda_{j}\right|<1$ for all of Ts eigenvalues $\lambda_{j}$. This latter condition implies that $\theta, \Delta t, \Delta x, \Delta z$ must be selected to satisfy ${ }^{5}$

$$
\theta \frac{4 \Delta t}{\phi_{M N N}}\left(\frac{k_{r_{M a x}}}{r_{w}^{2} \Delta x^{2}}+\frac{k_{z_{M a x}}}{H^{2} \Delta z^{2}}\right)<1,
$$

where $k_{r_{M a x}}$ and $k_{z_{M a x}}$ are upper bounds for horizontal and vertical permeability, respectively, and $\phi_{M I N}$ is a lower bound for porosity ${ }^{5}$. Reordering (10) as

$$
\boldsymbol{P}^{n+1}=(I-T) \boldsymbol{P}^{n}+T\left(\theta T\left(\boldsymbol{P}^{n}+\theta T\left(-\boldsymbol{P}^{n}+\theta T\left(\boldsymbol{P}^{n}+\theta T\left(-\boldsymbol{P}^{n}+\theta T(\ldots \ldots)\right)\right)\right)\right)\right)+\gamma
$$

$P^{n+1}$ is computed minimizing the number of operations.

Assuming that $\Delta x$ and $\Delta z$ are fixed, the largest $\Delta t$ is obtained when $\theta=0.25^{5}$.

\section{RESULTS}

\section{Numerical Analysis}

First, the 2-D simulator was tested by comparing its results against those of the analytical solution of a simplified 1-D case ${ }^{6}$ and the analytical solution of a two isolated layer reservoir ${ }^{1}$. The approximate early times analytical solution for the 1-D case ( $k_{r}$ is the constant horizontal permeability) is

$$
p_{w f}=p_{I N}-\frac{q_{T} \mu}{4 \pi k_{r} H}\left(\ln t-05772-\ln \left(\frac{\phi \mu c r_{w}^{2}}{4 k_{r}}\right)\right) \text {, }
$$

and for the two isolated layer reservoir $\left(k_{r 1}, k_{r 2}\right.$ and $h_{1}, h_{2}$ are the horizontal constant permeabilities and thicknesses of each layer) is

$$
p_{w f}=p_{I N}-\frac{q_{T} \mu}{4 \pi \frac{k_{r 1} h^{2}+k_{r 2} h_{2}}{h_{1}+h_{2}}\left(h_{1}+h_{2}\right)}\left(\ln t-05772-\frac{k_{r 1} \ln \ln \left(\frac{\phi_{1} \mu c r_{w 1}^{2}}{4 k_{r 1}}\right)+k_{r 2} h_{2} \ln \left(\frac{\phi_{2} \mu c_{r}^{2}}{4 k_{r 2}}\right)}{k_{r 1} h_{1}+k_{r 2} h_{2}}\right) .
$$

In both cases, the behavior of the numerical 2-D simulator coincides with the analytical solution. Besides, the results of the crossflow case (neglecting gravity) coincides with those obtained by Prijambodo et al $^{3}$.

Second, TSMF is compared with two iterative methods, ADI and Block-SOR, usually applied to solve multidimensional equations. Both methods, ADI and Block-SOR, have been adapted to this particular problem $^{5}$. We test a crossflow two layer reservoir with gravity effects. Horizontal permeabilities are $100 \mathrm{mD}$ and $10 \mathrm{mD}$ for each layer and vertical permeability is $10 \%$ of horizontal permeability. The three methods are compared taking $\theta=0.25$ and time step $0.25 \mathrm{~s}$. Reservoir data are shown in Table 1 . Total CPU time consumed to simulate 10 hours, using a personal computer PC 486 DX II with $16 \mathrm{MB}$ of RAM memory and $66 \mathrm{MHz}$, may be seen in Table 2. TSMF is the fastest technique, but the maximum allowed $\Delta t$ may be very low, depending upon the rock and fluid data (Eq. 11). On the other hand, blockSOR uses a large amount of computer time, but this time can be drastically reduced taking variable time increments. ADI is discarded for not presenting advantages over the other two techniques. The same results have been obtained with more layers ${ }^{5}$, where TSMF is even more convenient.

\section{Effects of vertical permeability and gravity on well performance}

Common reservoir data are shown in Table 1 . Three cases with different permeability arrangements are considered: 1- Equivalent single layer (homogeneous) reservoir of $H=20 \mathrm{~m}$ and $k_{r}=505 \mathrm{mD}$. 2- Isolated layers (commingled reservoir). It has two layers of $10 \mathrm{~m}$ which are separated by an impermeable shale of negligible thickness. They only communicate at the wellbore. Let us differentiate two subcases: 2a- 
$k_{r l}=10 \mathrm{mD}, k_{r 2}=1000 \mathrm{mD}$ (the less permeable layer on top) and $2 \mathrm{~b}-k_{r 1}=1000 \mathrm{mD}, k_{r 2}=10 \mathrm{mD}$ (the more permeable layer on top). 3- Crossflow reservoir. The same two layers as before, but they are communicated in the formation. The relationship between vertical and horizontal permeabilities in each layer can take values of $k_{r} / k_{r}=1,0.1,0.01$.

\begin{tabular}{|c|c|c|c|c|c|}
\hline \multicolumn{4}{|c|}{ TABLE 1: rock and fluid properties } & \multicolumn{2}{|c|}{ TABLE 2: total CPU time } \\
\hline oil compressibility, $c$ & $5 \times 10^{-9} \mathrm{~Pa}^{-1}$ & reservoir thickness, $H$ & $20 \mathrm{~m}$ & method & CPU [min] \\
\hline oil density, $\rho$ & $800 \mathrm{~kg} / \mathrm{m}^{3}$ & external radius, $r_{a}$ & $200 \mathrm{~m}$ & block-SOR & 23.99 \\
\hline oil viscosity, $\mu$ & $2 \times 10^{-3}$ Pa.s & wellbore radius, $r_{w}$ & $0.1 \mathrm{~m}$ & ADI & 1.25 \\
\hline initial pressure, $p_{I N}$ & $100 \times 10^{5} \mathrm{~Pa}$ & total flow rate, $q_{T}$ & $10^{-3} \mathrm{~m}^{3} / \mathrm{s}$ & SMF & 2.50 \\
\hline porosity, $\phi$ & 0.2 & $\begin{array}{l}\text { formation volume } \\
\text { factor, } B\end{array}$ & 1 & $\begin{array}{c}\text { block-SOR } \\
\text { (variable } \Delta t)\end{array}$ & 3.22 \\
\hline
\end{tabular}

Effects of vertical permeability. Fig. 1 shows the drawdown response for the three cases above mentioned. Gravity effects are neglected. The upper dash line corresponds to the equivalent single layer reservoir. The lower continuous line corresponds to the reservoir with two isolated layers. The curves are parallel to each other at early times. At late times, they are separated because the commingled reservoir feels the external boundary sooner. Dotted lines represent crossflow. At very early times, the flowing pressure of the crossflow system behaves as that of the two isolated layers system. At late times, it behaves as the equivalent single layer system. At the transitional period in between, the interlayer crossflow is dominant: the $p_{w j}(t)$ curves have an elongated " $\mathrm{S}$ " shape. At first, the slope of the curve decreases, reflecting the dominant contribution to production of the higher permeability layer. Then there is an inflection point and the slope increases again reaching the mean arithmetic permeability value of the equivalent single layer system. The point of departure and duration of the transitional period depend on the values of $k_{r} / k_{r 2}$ and $k_{z} / k_{r}$, as shown by Prijambodo et $\mathrm{al}^{3}$.

Effects of gravity on an homogeneous reservoir. If vertical permeability is zero the influence of gravity disappears in Eq.1. However, it still persists in the boundary condition at the well (Eq.4) . In Fig. 2, and also in Fig. 3 and 4, the flowing pressure is measured at mid-point of the upper layer face. Therefore, the curves $p_{w}(t)$ considering and neglecting gravity are completely parallel. The difference between them is $\rho g H / 4$.

Combined effects of vertical permeability and gravity, when the higher permeability $\left(k_{L}=1000 \mathrm{mD}\right)$ is at the bottom. During production, the more permeable layer is depleted faster. Therefore, a vertical pressure gradient is created. For crossflow reservoirs there are two driving forces: the vertical pressure gradient and gravity. In Fig. 3, they go in the same direction - the driving force is increased with respect to the case of nil gravity. The pressure responses of a crossflow reservoir with and without gravity effects are presented (Fig. 1 is superimposed on Fig. 3). When gravity is taken into account, the same three flow periods of Fig. 1 appear - but enlarged. In this particular case, there is a very important permeability contrast between the two layers. Therefore, nearly all production of the stratified system with isolated layers comes from the $1000 \mathrm{mD}$ layer. The sandface flowing pressure is controlled by that layer. Consequently, the difference between $p_{w f}$ curve of the commingled (isolated layers) system with gravity (Fig.3) and without gravity (Fig.1) is approximately $\rho g H / 2$.

Combined effects of vertical permeability and gravity, when the lower permeability $\left(\mathrm{k}_{\mathrm{r}}=10 \mathrm{mD}\right)$ is at the bottom. In Fig.4, the pressure responses of a crossflow reservoir with and without gravity effects are shown (Fig. 1 is superimposed on Fig. 4). In this case, the sandface flowing pressure is controlled by the upper layer of $1000 \mathrm{mD}$. As in Fig.3, the flowing pressure of the upper layer is shown. Therefore, results of the commingled (isolated layers) system with gravity (Fig.4) and without gravity (Fig.1) are practically equal. These results of the commingled (isolated layers) system present higher values than the flowing pressure curves of the equivalent single layer system considering gravity. Therefore, the crossflow system presents the three flow periods of Fig. 1 -but upside down. Let us notice that the vertical pressure gradient and the gravity forces go in opposite directions, being the latter greater than the former. If they were equal Fig. 4 would be a mirror image of Fig. 1 . 


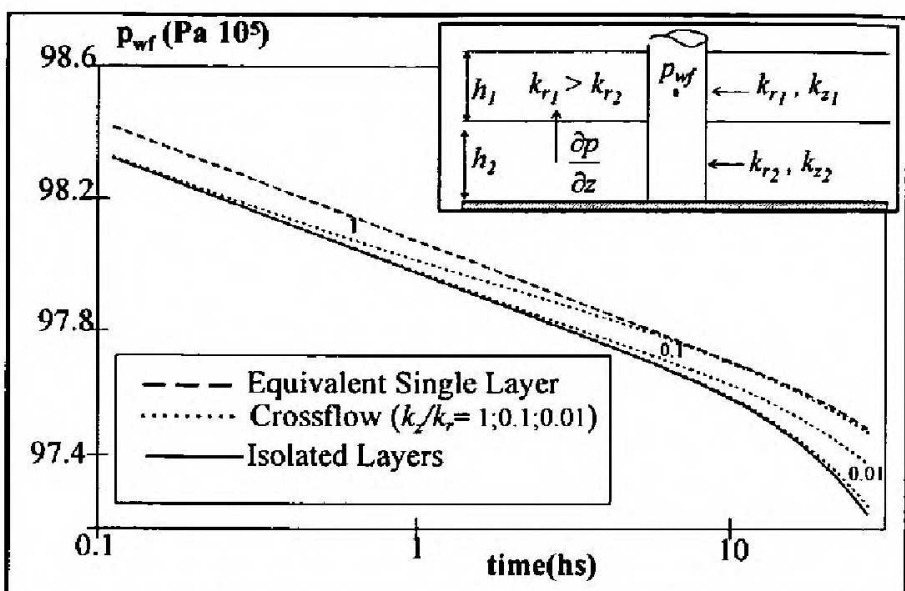

Fig. 1-Wellbore flowing pressure vs. time of the three reservoirs: equivalent single layer $\left(k_{r}=505 \mathrm{mD}\right)$, isolated layers $\left(k_{r l}=1000 \mathrm{mD}, k_{r 2}=10 \mathrm{mD}\right)$ and crossflow (different $k_{z} / k_{r}$ ). Gravity effects are neglected.

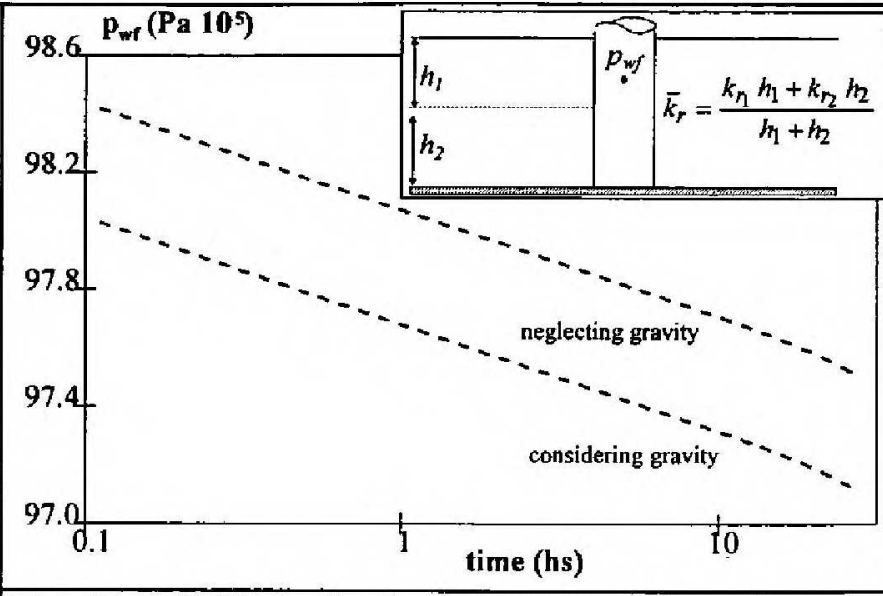

Fig. 2 - Wellbore flowing pressure vs. time from the equivalent single layer reservoir $\left(k_{r}=505 \mathrm{mD}\right)$, neglecting and considering gravity effects.

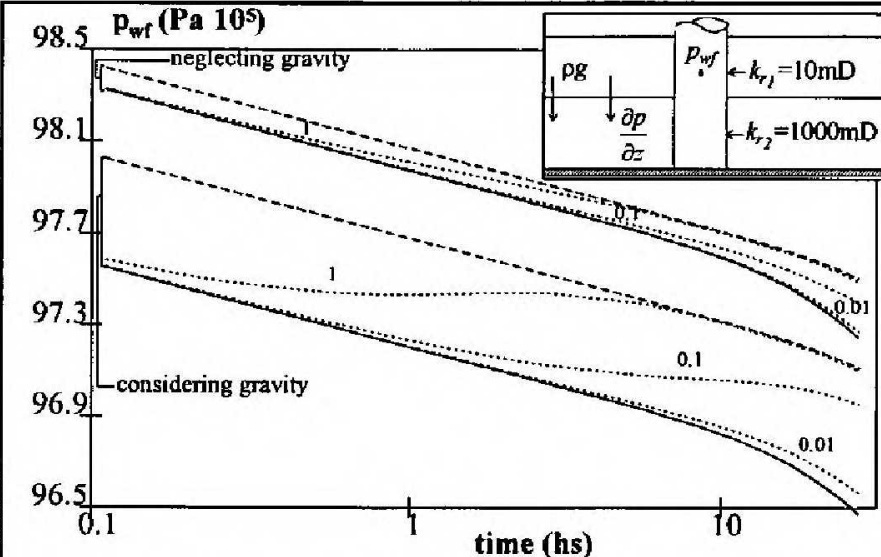

Fig. 3 - Wellbore flowing pressure vs. time from the upper layer (less permeable) of the three reservoirs -single layer, isolated layers and crossflow. Gravity effects are considered (below) and neglected (above).



Fig. 4 - Wellbore flowing pressure vs. time from the upper layer (more permeable) of the three reservoirs -single layer, isolated layers and crossflow. Gravity effects are considered (below) and neglected (above).

\section{CONCLUSIONS}

The equation that models the single phase flow of oil in a heterogeneous reservoir was solved in two dimensions $(r, z)$ using a family of finite difference schemes. This family depends on one parameter $\theta$. The resulting system of linear equations is solved by a method based on Taylor Series of Matrix Functions which is applied to this problem for the first time. TSMF is compared with two iterative methods, ADI and Block-SOR. The simulator is applied to analyze the transient pressure behavior of a drawdown test performed in a two layer reservoir with crossflow and gravity effects. Numerical and physical conclusions are:

1. TSMF is the fastest technique, for complex situations like crossflow in multilayered formations, using adequate values of $\theta$ and $\Delta t$, but the time step $\Delta t$ must remain small because of the convergence condition $^{5}$. Therefore, TSMF is recommended when a short period of time must be simulated.

2. Block-SOR converges with longer values of $\Delta t$. Yet it consumes a large amount of CPU time which can be reduced using a variable time step. Accordingly, Block-SOR is suitable for long simulations and applying variable time steps.

3. Combining TSMF for early times and Block-SOR with variable $\Delta t$ for later times, the most efficient algorithm is obtained. ADI is discarded because it has no advantages over the other two techniques.

4. The pressure response of crossflow reservoirs lies between the response of the equivalent single layer system and that of the isolated layer system. It can be divided into three flow periods: At very early times, it behaves as if the layers were isolated. At a transitional period, the pressure response depends on 
the interlayer flow; as vertical permeability increases the transitional period starts earlier. At later times, the reservoir behaves as an equivalent single layer homogeneous system ${ }^{3}$.

5. When gravity effects are added, the pressure response is affected by the order of the layers. If the lower layer is the more permeable one, the driving forces for crossflow are increased and the gap between the response of the equivalent single layer system and that of the isolated layer system is widened. The same three flow periods above mentioned appear, but enlarged.

6. If the upper layer is the more permeable, the vertical pressure gradient and the gravity forces go in opposite directions. Hence, the gap is lessened. When gravity forces are greater than the vertical pressure gradient, the pressure response of the isolated layer system presents higher values than that corresponding to the equivalent single layer system. Therefore, the same three flow periods occur, but upside down.

Acknowledgments - We are indebted to the University of Buenos Aires for supporting this work and to Larry $W$. Lake of the University of Texas at Austin for useful suggestions. M.S.Bidner is a Research Fellow at the Consejo Nacional de Investigaciones Cientificas y Técnicas de Argentina.

\section{NOMENCLATURE}

$B=$ formation volume factor (res $\mathrm{m}^{3} /$ stock-tank $\mathrm{m}^{3}$ )

$c=$ oil compressibility $\left(\mathrm{Pa}^{-1}\right)$

$g=$ aceleration of gravity $\left(\mathrm{m} / \mathrm{s}^{2}\right)$

$H=$-reservoir thickness (m)

$I=$ Identity matrix

$k_{r}=$ horizontal permeability (mD)

$k_{z}=$ vertical permeability $(\mathrm{mD})$

$\bar{N}=$ vector dimension

$p=$ pressure $(\mathrm{Pa})$

$p_{I N}=$ initial pressure $(\mathrm{Pa})$

$p_{w p}=$ wellbore flowing pressure $(\mathrm{Pa})$

$q_{T}=$ total flow rate $\left(\mathrm{m}^{3} / \mathrm{s}\right)$

r-radius (m)

$r_{e}=$ external radius $(\mathrm{m})$

$r_{\mathrm{w}}=$ wellbore radius $(\mathrm{m})$

$t=$ time (s)

$T=$ matrix of the numerical scheme

$x_{D}=$ auxiliary variable

$z=$ vertical coordinate

\author{
GREEK LETTERS \\ $\Delta t=$ time discretization step \\ $\Delta \mathrm{x}=$ space increment $(\mathrm{x}$ coordinate) \\ $\Delta \mathrm{z}=$ space increment ( $\mathrm{z}$ coordinate) \\ $\phi=$ porosity (fraction) \\ $\lambda=$ eigenvalue \\ $\mu=$ viscosity (Pa.s) \\ $\theta=$ parameter of finite difference schemes \\ $\rho=$ density $\left(\mathrm{kg} / \mathrm{m}^{3}\right)$ \\ SUBSCRIPTS \\ $D=$ dimensionless \\ $i=$ corresponding to $\mathrm{i}^{\text {th }}$ space grid \\ $j$-corresponding to $j^{\text {th }}$ space grid \\ $M A X=$ maximum value \\ $M I N=$ minimum value \\ SUPERSCRIPTS \\ $n=$ corresponding to $\mathrm{n}^{\text {th }}$ time step
}

\section{REFERENCES}

1. Lefkovits, H. C., Hazebroek, P., Allen, E. E. and Matthews, C. S., 1961. "A Study of the Behavior of Bounded Reservoirs Composed of Stratified Layers". Society of Petroleum Engineers Journal, 1(1), 43-58.

2. Russell, D. G. and Prats, M., 1962. "Performance of Layered Reservoirs with Crossflow-Single-CompressibleFluid Case". Society of Petroleum Engineers Journal, Trans., AIME, 225, 53-67.

3. Prijambodo, R, Raghavan, R. and Reynolds, A., 1985. "Well Test Analysis for Wells Producing Layered Reservoirs with Crossflow". Society of Petroleum Engineers Journal, 25(3), 380-396.

4. Savioli, G. B., Bidner, M. S. and Jacovkis, P. M., 1996. "Statistical Analysis of Heterogeneities and their Effect on Build-Up and Draw-Down Tests". Journal of Petroleum Science \& Engineering, 15, 45-55.

5. Savioli, G. B., 1996. "Modelos Matemáticos en Ingenieria de Reservorios". Ph.D. Thesis, Department of Mathematics, School of Exact Sciences, University of Buenos Aires.

6. Savioli, G. B., Jacovkis P. M. and Bidner, M. S., 1997. "Stability Analysis and Numerical Simulation of 1-D and 2-D Radial Flow towards an Oil Well". Computers Math. Applic. 33 (3), 121-135.

7. Darderes, E., Vampa, V., Sorarrain, O. and Bidner, M. S., 1988. "Gas Well Testing Analysis Using FiniteDifference Models and Optimization Techniques". Revue de L'Institut Francais du Pétrole, 43(3), 371-387.

8. Savioli, G. B. and Bidner, M. S., 1994. "Aplicación del método inverso al análisis de ensayos de pozos petroliferos". Revista Internacional de Métodos Numéricos para Cálculo y Diseño en Ingeniería, 10(1), 3-21.

9. Savioli, G. B. and Bidner, M. S., 1994. "Comparison of Optimization Techniques for Automatic History Matching". Journal of Petroleum Science \& Engineering, 12,25-35. 